\title{
A Mathematical Note on All-Productive, All-Engaging and Related Systems
}

\author{
Giorgio Giorgi ${ }^{1}$ \\ ${ }^{1}$ Faculty of Economics, Via S. Felice, 5-27100 Pavia, Italy \\ Correspondence: Giorgio Giorgi, Faculty of Economics, Via S. Felice, 5-27100 Pavia, Italy. E-mail: \\ ggiorgi@eco.unipv.it
}

Received: October 8, 2013 Accepted: November 27, 2013 Online Published: December 24, 2013

doi:10.5539/jmr.v6n1p1

URL: http://dx.doi.org/10.5539/jmr.v6n1p1

\begin{abstract}
The purpose of this paper is to apply some specific results of matrix analysis to the study of the solvability properties of linear joint production models, both for the non- square case and for the square case.
\end{abstract}

Keywords: joint production models, linear models, all-productive systems, all-engaging systems, Sraffa joint production models, $S$-matrices, $K$-matrices

\section{Introduction}

Linear joint production models, described by systems of linear equations, have received a considerable attention, especially after the publication of the famous Sraffa's book (1960), where pure joint production models are treated in the second part, dedicated to "Multiple-Product Industries and Fixed Capital". However, a purely mathematical analysis of these (and similar) models is less abundant. For instance, without pretension of completeness, we can quote Abraham-Frois and Berrebi (1976, 1997), Bidard (1996, 2004), Bidard and Erreygers (1998), Manara (1968), Peris and Villar (1993), Giorgi and Magnani (1978), Lippi (1979), Kurz and Salvadori (1995), Schefold (1971, 1976, 1978a, 1978b, 1989).

The literature on linear joint production models based on inequalities (essentially the famous von Neumann growth model, its variants and generalizations) is in general more mathematically oriented. Here we are concerned only with some formal properties of linear joint production models described by equations and by a pair of matrices $(A, B)$. We shall benefit from some specific results of matrix algebra and only occasionally we shall give economic interpretations or considerations.

This paper may be considered as a complement and prosecution of the papers of Bidard (1996) and Bidard and Erreygers (1998). It is organized as follows.

Section 2 is concerned with general joint production models, i.e. models where the matrices $A, B$ are not necessarily square.

Section 3 is concerned with the case, more fruitful at least from the mathematical point of view, of square matrices $A, B$, i.e. with the case considered by Sraa.

In the present paper the following notations will be used. All matrices are real and are denoted by capital letters: $A \in \mathbb{R}^{m \times n}$ is a matrix of $m$ rows and $n$ columns; its $i$-th row is denoted by $A_{i}, i=1, \ldots, m$, whereas its $j$-th column is denoted by $A^{j}, j=1, \ldots, n$. The vectors of $\mathbb{R}^{n}$ are denoted by lower-case letters. The symbol [0] is used to denote a zero vector or a zero matrix. The notation $A^{T}$ stands for the transpose of $A, x^{T}$ is the transpose of the vector $x, I$ is the identity matrix. The notations $A>[0], A \geqq[0], A \geq[0]$ are used to denote, respectively, a positive matrix, i. e. $a_{i j}>0, \forall i, j$; a nonnegative matrix, i. e. $a_{i j} \geqq 0, \forall i, j$; a semipositive matrix, i. e. $A \geqq[0], A \neq[0]$. $A<[0], A \leqq[0], A \leq[0]$ are defined in a similar way. The same convention is used to compare vectors with the zero vector [0] and the same convention is used to compare two matrices (of the same order) or two vectors (of the same dimension).

We consider a classical joint production model, described by equations and involving $m$ commodities and $n$ processes (or industries or activities). The $j$-th process uses the inputs described by $A^{j}$ and produces the outputs 
described by the corresponding $j$-th column $B^{j}$. So, the data of the model are described by the pair $(A, B)$. Obviously, we have $A \geqq[0], B \geqq[0]$, but we accept also two assumptions introduced by Kemeny, Morgenstern and Thompson (1956), i.e.

$$
\begin{gathered}
A^{j} \geq[0], \forall j=1, \ldots, n ; \\
B_{i} \geq[0], \forall i=1, \ldots, m .
\end{gathered}
$$

Relation (1) simply says that every process uses at least one positive input ("no free lunch"); relation (2) says that each commodity can be produced by some activity (we say also, if (2) holds, that the model is complete). It is also convenient to accept the assumption

$$
B^{j} \geq[0], \forall j=1, \ldots, n,
$$

which says that every process yields at least one positive output (there is no "pure waste" process activated).

The vector $x \in \mathbb{R}^{n}, x \geqq[0]$, describes the (column) vector of activity intensities and the vector $p \in \mathbb{R}^{m}, p \geqq[0]$, describes the (row) vector of prices. If $m=n$ the model is called square. The quantities $A x, B x,(B-A) x, p(B-A)$ have an obvious economic meaning. We point out that some definitions and results considered in the sequel, have a mathematical meaning independent from the assumptions made on $A$ and $B$.

Definition 1 The model $(A, B)$ is productive if there exists an activity vector $x \geq[0]$ such that $y=(B-A) x>[0]$. In mathematical terms, this is equivalent to: the matrix $(B-A)$ belongs to the $S$-class, i.e. the system

$$
\left\{\begin{array}{l}
(B-A) x>[0] \\
x \geq[0]
\end{array}\right.
$$

admits a solution $x$. The $S$-class of matrices or class of $S$-matrices was introduced by Stiemke in 1915 and studied in detail by Fiedler and Pták (1966), together with other classes of matrices, defined below.

Definition 2 Let a matrix $A \in \mathbb{R}^{m \times n}$ be given.

(i) The matrix $A$ is an $S_{0}$-matrix if the system

$$
\left\{\begin{array}{l}
A x \geqq[0] \\
x \geq[0]
\end{array}\right.
$$

admits a solution $x$.

(ii) The $S_{0}$-matrix is an $M$-matrix if either $n=1$ or $n>1$ and no matrix obtained from $A$ by omitting at least one column is an $S_{0}$-matrix. (The terminology is due to Fiedler \& Pták, 1966, but this class must be not confused with the class of $M$-matrices of Berman \& Plemmons, 1976; Plemmons, 1977; Poole \& Boullion, 1974, and other authors).

In the present paper the letters $S, S_{0}, M$ will also be used to denote the classes of the corresponding matrices. We now give the main characterizations of the classes previously considered. See Fiedler and Pták (1966) and Giorgi and Zuccotti (2013).

Theorem 1 Let $A \in \mathbb{R}^{m \times n}$; the following properties are equivalent characterizations of the $S$-class.

(a) The system $A x>[0]$ admits a solution $x \geqq[0]$.

(b) The system $A x>[0]$ admits a solution $x \geq[0]$.

(c) The system $A x>[0]$ admits a solution $x>[0]$.

(d) For every vector $p \geq[0]$, at least one component of the vector $p A$ is positive.

(e) It holds

$$
p \geq[0] \Longrightarrow p A \not[0] .
$$

(f) It holds

$$
p A \leqq[0] \Longrightarrow p \nsupseteq[0] .
$$

( $g$ ) For every vector $p \leq[0]$, at least one component of $p A$ is negative.

(h) It holds

$$
p \leq[0] \Longrightarrow p A \nsupseteq[0] .
$$


(i) It holds

$$
p A \geqq[0] \Longrightarrow p \not \leq[0] .
$$

Theorem 2 Let $A \in \mathbb{R}^{m \times n}$; the following properties are equivalent characterizations of the $S_{0}$-class.

(a) The system $A x \geqq[0]$ admits a solution $x \geq[0]$.

(b) For every vector $p \geqq[0]$, at least one component of the vector $p A$ is nonnegative.

(c) It holds

(d) $(A+B) \in S$, for every $B>[0]$.

$$
p \geqq[0] \Longrightarrow p A \nless[0] .
$$

Theorem 3 Let $A \in \mathbb{R}^{m \times n}$; the following properties are equivalent characterizations of the $M$-class.

(a) Either $A \in S_{0}$ and A has only one column, or $A \in S_{0}$, but no matrix obtained from A by omitting at least one column is an $S_{0}$-matrix.

(b) $A \in S_{0}$ and the system

$$
\left\{\begin{array}{l}
A x \geqq[0] \\
x \geq[0]
\end{array}\right.
$$

admits only solutions $x>[0]$.

(c) $A \in S_{0}$ and for every solution $x \neq[0]$ of $A x \geqq[0]$, it holds either $x>[0]$ or $x<A x=[0]$.

The inclusions $S \subset S_{0}$, and $M \subset S_{0}$ are strict. For other considerations see Giorgi and Zuccotti (2013).

\section{General Joint Production Models}

We point out that, from a theoretical point of view, any arbitary matrix can be written as a difference of two nonnegative matrices ("positive splitting"), but here $A$ and $B$ are given, they are the data of our economic model.

Definition 3 The model $(A, B)$ is profitable if there exists a price vector $p \geq[0]$ such that $v=p(B-A)>[0]$.

The model is therefore profitable if the system

$$
\left\{\begin{array}{l}
(B-A)^{T} p^{T}>[0] \\
p^{T} \geq[0]
\end{array}\right.
$$

has a solution $p$, i.e. if $(B-A)^{T} \in S$, or, with an evident meaning of the notation $S^{T}$, if $(B-A) \in S^{T}$.

We have to remark that the two properties of productivity and profitability are compatible, but independent properties. In other words the classes $S$ and $S^{T}$ are not disjoint, but have only a partial overlapping. When the model is square and $B=I$ ("simple production"), then $(B-A)$ becomes $(I-A)$, with $(I-A) \in Z, Z$ being the class of square matrices $Z=\left[z_{i j}\right]$ with $z_{i j} \leqq 0, \forall i \neq j$. In this case productivity coincides with profitability. This is a classical result of the theory of (square) $K$-matrices (in the terminology of Fiedler \& Pták, 1962) or $M$-matrices, in the terminology of other authors, such as the ones previously quoted in Definition 2, at the point (ii). In the general case we can formulate the following test of productivity and profitability of a model $(A, B)$.

Theorem 4 (i) The model $(A, B)$ is productive if and only if, for any price vector $p \geq[0]$, there exists an activity (in general varying with the choice of $p$ ) such that the corresponding net value is positive:

$$
p \geq[0] \Longrightarrow \exists j: p(B-A)^{j}>0 .
$$

(ii) The model $(A, B)$ is profitable if and only if, for any activity vector $x \geq[0]$, there exists a commodity (in general varying with the choice of $x$ ) such that the corresponding net production is positive:

$$
x \geq[0] \Longrightarrow \exists i:(B-A)_{i} x>0 .
$$

The part $(i)$ of the previous theorem is nothing but the characterization $(d)$ of $S$-matrices in Theorem 1 . We give, for the reader's convenience, a complete proof of the theorem. First we recall the Ville theorem of the alternative (see, e.g., Cottle, Pang, \& Stone, 1992).

Lemma 1 Let $A \in \mathbb{R}^{m \times n}$ be given. The system

$$
A x>[0], x>[0]
$$


has a solution if and only if the system

$$
y A \leqq[0], \quad y \geq[0]
$$

has no solution.

In terms of the classes $S$ and $S_{0}$, the Ville theorem of the alternative can therefore be described by the following equivalence

$$
(A \in S) \Longleftrightarrow\left(\left(-A^{T}\right) \notin S_{0}\right) .
$$

Proof of Theorem 4. Thanks to Lemma $1,(B-A) \in S$ if and only if $\left[-(B-A)^{T}\right] \notin S_{0}$. This means that the system

$$
\left\{\begin{array}{l}
{\left[-(B-A)^{T}\right] p^{T} \geqq[0]} \\
p^{T} \geq[0]
\end{array}\right.
$$

i.e. the system

$$
\left\{\begin{array}{l}
p(B-A) \leqq[0] \\
p \geq[0]
\end{array}\right.
$$

has no solution. Therefore $(i)$ is proved. In a symmetric way, $(B-A)$ is profitable if and only if $(B-A)^{T} \in S$, i.e., thanks to the same theorem of the alternative, if and only if $\left[-(B-A)^{T}\right]^{T} \notin S_{0}$, i.e. $[-(B-A)] \notin S_{0}$. This means that the system

$$
\left\{\begin{array}{l}
{[-(B-A)] x \geqq[0]} \\
x \geq[0],
\end{array}\right.
$$

i.e. the system

$$
\left\{\begin{array}{l}
(B-A) x \leqq[0] \\
x \geq[0]
\end{array}\right.
$$

has no solution. Therefore, (ii) is proved.

Obviously, the principal relevance of the above tests relies on the possibility to detect non-productive models and non-profitable models, rather than productive models and profitable models.

We recall that $(B-A) \in M$ if $(B-A) \in S_{0}$ and, moreover, it holds

$$
(x \geq[0],(B-A) x \geqq[0]) \Longrightarrow x>[0] .
$$

(See Theorem 3).

If $(B-A) \in M,(B-A)$ not square, the number of processes is always less than the number of commodities $(n<m)$, thanks to Property 3.8 of Fiedler and Pták (1966). Moreover, always under the said assumptions, if the columns $(B-A)^{j}, j=1, \ldots, n$, are linearly independent, then the pair $(A, B)$ is profitable and quasi-productive, i.e. the system

$$
\left\{\begin{array}{l}
(B-A) x \geq[0] \\
x \geqq[0]
\end{array}\right.
$$

has a solution.

We can note that relation (4) establishes a sort of connectedness of the model $(A, B)$ : if a sector produces a positive output, the other sectors are also "obliged" to produce positive outputs. We give therefore the following definition.

Definition 4 The model $(A, B)$ is said to have the connectedness property if relation (4) holds.

The economic literature has reserved more attention, especially after the publication of the famous book of Sraffa (1960), to the case of uniform distribution of the net production and uniform distribution of the net value (i.e. the value of the net production). We have, respectively, the system

$$
\left\{\begin{array}{l}
(B-A) x=g A x+c \\
x \geq[0], g \geqq 0,
\end{array}\right.
$$


in the unknown pair $(x, g)$, where $c \geqq[0]$ is the vector of the consumption basket and $g$ is the uniform growth rate, and the system

$$
\left\{\begin{array}{l}
p(B-A)=r p A+w l \\
p \geq[0], r \geqq 0, w \geqq 0,
\end{array}\right.
$$

in the unknown triplet $(p, r, w)$, where $r$ is the uniform profit rate, $l>[0]$ is the vector of direct labour requirements, and $w \geqq 0$ is the real wage. We point out that a model $(A, B)$ can be productive, but the system (5) admits no solution; similarly, $(A, B)$ can be profitable, but the system (6) admits no solution. It is possible to construct numerical examples. Take, e.g., the model

$$
A=\left[\begin{array}{lll}
3 & 3 & 1 \\
1 & 3 & 6 \\
2 & 1 & 1
\end{array}\right], \quad B=\left[\begin{array}{ccc}
1 & 0 & 2 \\
3 & 8 & 5 \\
4 & 3 & 15
\end{array}\right], \quad c=\left[\begin{array}{l}
1 \\
1 \\
0
\end{array}\right]
$$

The model $(A, B)$ is productive, but the system (5) admits no solution.

Mangasarian (1971) in his important paper on the generalization of the Perron-Frobenius theorem to the matrix pair (or "matrix pencil") $(A, B)$, with $A$ and $B$ of order $(m, n)$ and not necessarily nonnegative, has introduced the following assumption:

$$
\exists H \in \mathbb{R}^{n \times n}, H \geqq[0]: A=B H .
$$

This assumption does not assure, however, that the model $(A, B)$ is productive, nor profitable. Mangasarian shows that (7) is equivalent to the implication

$$
p B \geqq[0] \Longrightarrow p A \geqq[0],
$$

of evident and acceptable economic meaning for $p \geqq[0]$, but with doubtful economic meaning if $p$ has elements of opposite sign.

We can introduce also the following variants of Mangasarian's assumption (7):

$$
\exists H \in \mathbb{R}^{m \times m}, H \geqq[0]: A=H B
$$

which is equivalent to the implication

$$
B q \geqq[0] \Longrightarrow A q \geqq[0] .
$$

Fujimoto and Krause (1988) call this last implication "non-dominance property".

$$
\exists H \in \mathbb{R}^{m \times m}, H \geqq[0]: B=H A
$$

which is equivalent to the implication

$$
\begin{gathered}
A q \geqq[0] \Longrightarrow B q \geqq[0] . \\
\exists H \in \mathbb{R}^{n \times n}, H \geqq[0]: B=A H
\end{gathered}
$$

which is equivalent to the implication

$$
p A \geqq[0] \Longrightarrow p B \geqq[0] .
$$

If we impose on the matrix $H$ some further properties, then Mangasarian's assumptions can assure productivity or profitability. Indeed, under the assumptions (1), (2), it is quite easy to prove that, if $\lambda^{*}(H)<1, \lambda^{*}(H)$ being the Frobenius root of $H \geqq[0]$ (see Berman \& Plemmons, 1976; Gantmacher, 1959), the model where (7) holds is productive, but not necessarily profitable, whereas the model where (8) holds is profitable, but not necessarily productive. If $H$ is indecomposable (see Definition 6 in Section 3) and $\lambda^{*}(H)>1$, the model where (9) holds is profitable, but not necessarily productive. Under the same assumptions on $H^{T}$, the model where (10) holds is quasi-productive, but not necessarily profitable.

Other results, under Mangasarian's assumptions, are obtained by Fujimoto and Krause (1988). For example, taking some results of Mangasarian into account, it is easy to show that if the model $(A, B)$ satisfies relation $(8)$ and each row $A_{i}$ is semipositive, then the system

$$
\left\{\begin{array}{l}
p B=(1+r) p A \\
p \geq[0],(1+r)>0
\end{array}\right.
$$


has a solution. Note that this result does not assure the positiveness of $r$. Another result of Fujimoto and Krause is the following one: if the model $(A, B)$ satisfies (8) and is productive, then the system (11) has a solution where $r>0$.

Another property of a general linear joint production system, stronger than productivity, is given in the following definition.

Definition 5 The model $(A, B)$ is said to be strongly solvable (in the terminology of Peris \& Villar, 1993) or has the adjustment property (in the terminology of Bidard \& Erreygers, 1998) if for every vector $y \geq[0]$ the system

$$
\left\{\begin{array}{l}
(B-A) x=y \\
x \geq[0]
\end{array}\right.
$$

admits solution.

In economic terms: for every choice of a semipositive vector of net productions, described by the vector $y$, there exists a vector $x \geq[0]$ (obviously $x$ may vary with the choice of $y$ ) which yields exactly those net productions. We shall give in the next Section further insights on this property and its relationships to the productivity property.

\section{Square Joint Production Models}

In this section we assume that the matrices $A$ and $B$ are square, of order $n$. For economic reasons it is convenient to assume again (1), (2) and (3), even if several mathematical results hold also without these assumptions. We recall that joint production models with $A$ and $B$ square were considered in the second part of the book of Piero Sraffa (1960) and that for this case the economic literature is quite abundant (see, e.g., Bidard, 2004; Schefold, 1989, and the references quoted in these books). The following definitions are taken from Schefold (1971, 1989).

Definition 6 The model $(A, B)$ is called "all-productive" if $(B-A)^{-1} \geq[0]$; the model is called "all-engaging" if $(B-A)^{-1}>[0]$.

Obviously, if the model is all-productive, then it is also productive and profitable. The converse does not hold, as it can be shown by simple numerical examples, e.g. by choosing

$$
A=\left[\begin{array}{lll}
3 & 3 & 1 \\
1 & 3 & 6 \\
2 & 1 & 1
\end{array}\right], \quad B=\left[\begin{array}{lll}
1 & 0 & 2 \\
3 & 8 & 5 \\
4 & 3 & 5
\end{array}\right] \text {. }
$$

The following result was obtained by Bidard and Erreygers (1998), but our proof is more direct and simpler.

Theorem 5 The square model $(A, B)$ is strongly solvable if and only if $(B-A)$ is nonsingular and it holds $(B-A)^{-1} \geq$ [0], i.e. the model is all-productive.

Proof. $(\Longleftarrow)$. We have that for any $y \geq[0]$ the unique solution of the system $(B-A) x=y$ is given by the vector $x=(B-A)^{-1} y \geq[0]$ : indeed, it cannot be $x=(B-A)^{-1} y=[0]$, as then it would be [0] $=(B-A) x=y \geq[0]$, which is a contradiction.

$(\Longrightarrow)$. Let $e^{i}, i=1, \ldots, n$, be the $n$ canonical unit vectors of $\mathbb{R}^{n}$, i.e. $e^{i}=[0,0, \ldots, 1, \ldots, 0]^{T}$.

By assumptions, there exist $n$ semipositive vectors $y^{i} \geq[0], i=1, \ldots, n$, such that $(B-A) y^{i}=e^{i}$. If we consider the matrix $Y=\left[y^{1}|\ldots| y^{n}\right]$, we have $(B-A) Y=I$ and therefore $(B-A)^{-1}=Y \geq[0]$.

A classical result of Collatz (1966) shows that an arbitrary matrix $M$ has a semipositive inverse $M^{-1} \geq[0]$ if and only if

$$
M x \geqq[0] \Longrightarrow x \geqq[0],
$$

i.e. if $M$ is of monotone kind. In other words, the above implication is equivalent to: $M^{-1}$ exists and $M^{-1} \geq[0]$.

Therefore, we can also say that the square model $(A, B)$ is all-productive if and only if $(B-A)$ is of monotone kind. This property has also an evident economic interpretation. Moreover, we can note that the square model $(A, B)$ is all-productive if and only if there exists a semipositive and nonsingular matrix $V \in \mathbb{R}^{n \times n}$ such that

$$
x=V y, \quad p=v V,
$$

where $y=(B-A) x, v=p(B-A)$ for all $x$ and for all $p$. That is, $V$ is such that it transforms the vector of the net productions $y$ into the activity vector $x$ and the vector of the unitary net values $v$ into the price vector $p$. In other 
words $V$ solves the following two equivalent systems:

$$
\begin{aligned}
& V B=I+V A \\
& B V=I+A V .
\end{aligned}
$$

It may be interesting to put into evidence an economic interpretation of the columns of the matrix $(B-A)^{-1}$. Let us denote by $V$ the matrix $(B-A)^{-1}$ and by $V^{i}$ the $i$-th column of $V$. As $V^{-1} V=I$, it holds $V^{-1} V^{i}=e^{i}$. This means that $V^{i}$ describes the vector of activity intensities necessary to produce one unit of the $i$-th commodity and zero unit of all other $(n-1)$ commodities. For a discussion of the economic meaning of the matrix $A(B-A)^{-1}$ the reader may consult Pasinetti (1973).

Theorem 6 Let a square model $(A, B)$ be given. Then the following conditions are equivalent:

(i) The model is positively strongly solvable, i.e. for every vector $y \geq[0]$, the system

$$
\left\{\begin{array}{l}
(B-A) x=y \\
x>[0]
\end{array}\right.
$$

admits solution.

(ii) $(B-A)$ is nonsingular and it holds $(B-A)^{-1}>[0]$.

(iii) The model is strongly solvable and has the connectedness property (4), i.e.

$$
(x \geq[0],(B-A) x \geqq[0]) \Longrightarrow x>[0] .
$$

Proof. $(i) \Longleftrightarrow(i i)$. The proof is similar to the one of Theorem 5.

(ii) $\Longrightarrow($ iii $)$. Being $(B-A)^{-1}>[0]$, the model is strongly solvable; moreover, $x \geq[0]$ and $(B-A) x \geqq[0]$, the matrix $(B-A)$ being nonsingular, imply that $y=(B-A) x \geq[0]$. Since $(B-A)^{-1}>[0], x=(B-A)^{-1} y>[0]$, therefore the model has also the connectedness property.

(iii) $\Longrightarrow($ ii $)$. As the model $(A, B)$ is strongly solvable, then $(B-A)^{-1} \geq[0]$. Let $C^{i} \geq[0]$ be the $i$-th column of $C=(B-A)^{-1} \geq[0]$ and let $e^{i}, i=1, \ldots, n$, be the $i$-th canonical unit vector of $\mathbb{R}^{n}$. As $(B-A) C^{i}=e^{i} \geq[0]$, thanks to the connectedness property of the model, it must hold $C^{i}>[0]$ and therefore $C=(B-A)^{-1}>[0]$.

A result similar to Theorem 6 was given by Fiedler and Pták (1966), by means of properties of the $M$-matrices.

Theorem 7 The following two conditions are equivalent:

(i) $(B-A)$ is nonsingular and $(B-A) \in M$;

(ii) $(B-A)^{-1}>[0]$, i.e. the model $(A, B)$ is all-engaging.

Yet another similar characterization of all-engaging systems has been given by Bidard (1996), where however, the nonsingularity requirement in $(i)$ is omitted. In the proof of his result this author claims that the connectedness property (4) implies the nonsingularity of $(B-A)$, but this is false, as shown by the following example.

$$
(B-A)=\left[\begin{array}{cc}
-1 & 1 \\
1 & -1
\end{array}\right] ; \quad(B-A)\left[\begin{array}{l}
\alpha \\
\beta
\end{array}\right]=[0] \Longrightarrow \alpha=\beta
$$

Being $\alpha=\beta=0$ excluded, it will be $\alpha=\beta>0$. Then the model has the connectedness property, but $(B-A)$ is singular.

We note that $(B-A)$ can be all-engaging also in the case $(B-A) \notin Z$ : take, e.g., the matrices

$$
A=\left[\begin{array}{ccc}
3 / 2 & 1 & 4 \\
0 & 3 & 1 \\
4 & 2 & 4
\end{array}\right], \quad B=\left[\begin{array}{ccc}
0 & 2 & 5 \\
1 & 2 & 2 \\
5 & 3 & 5 / 2
\end{array}\right]
$$

In this case $(B-A)^{-1}>[0]$, but $(B-A) \notin Z$ (and also every permutation of its lines gives a matrix not in the $Z$-class). Obviously, if $(B-A) \in Z$ and $(A, B)$ is productive (i.e. $(B-A) \in S)$, then $(A, B)$ is all-productive, thanks to a well-known characterization of the $K$-matrices. We recall that a square matrix $H$ belongs to $K$ if and only if $H \in Z \cap S$; this is equivalent to: $H \in Z$ and $H^{-1} \geq$ [0]: see, e.g., Berman and Plemmons (1976), Magnani and Meriggi (1981). 
We now recall the concept of indecomposable (square) matrix, in order to point out the ralationships beteween the connectedness property and the indecomposability property.

Definition 7 A matrix $A \in \mathbb{R}^{n \times n}$, with $n \geqq 2$, is said to be decomposable orreducibleif there exists a permutation matrix $\Pi$, of order $n$, and an integer $k, 0<k<n$, such that

$$
A=\Pi\left[\begin{array}{cc}
A_{11} & A_{12} \\
{[0]} & A_{22}
\end{array}\right] \Pi^{T}
$$

with $A_{11} \in \mathbb{R}^{k \times k}, A_{22} \in \mathbb{R}^{(n-k) \times(n-k)}$, i.e. $A_{11}$ and $A_{22}$ square matrices. If $A$ is not decomposable, it is called indecomposable orirreducible.

We recall that a permutation matrix $\Pi$ is a matrix obtained from the identity matrix $I$ by means of the same permutations of its rows and columns. The matrix $\Pi$ is orthogonal, i.e. $\Pi^{-1}=\Pi^{T}$. It can be proved (see, e.g., Berman \& Plemmons, 1976) that the following conditions are equivalent:

(i) $A$ is an indecomposable nonnegative square matrix of order $n$.

(ii) $(I+A)^{(n-1)}>[0]$, where $A^{(k)}$ is the $k$-th power of $A$.

(iii) $I+A+A^{(2)}+A^{(3)}+\ldots+A^{(n-1)}=I+\sum_{k=1}^{n-1} A^{(k)}>[0]$.

(iv) $A+A^{(2)}+A^{(3)}+\ldots+A^{(n-1)}+A^{(n)}=\sum_{k=1}^{n} A^{(k)}>[0]$.

Lemma 2 Let $A \in \mathbb{R}^{n \times n}$ and let $A$ be nonsingular. Then $A$ is decomposable if and only if $A^{-1}$ is decomposable.

Proof. It is sufficient to remark that if $A$ is nonsingular and decomposable, then its inverse takes the form

$$
A^{-1}=\Pi\left[\begin{array}{cc}
A_{11}^{-1} & -A_{11}^{-1} A_{12} A_{22}^{-1} \\
{[0]} & A_{22}^{-1}
\end{array}\right] \Pi^{T} .
$$

Theorem 8 Let $(A, B)$ be a square linear joint production model. Let $(A, B)$ be strongly solvable and assume that $(A, B)$ has the connectedness property. Then the matrix $(B-A)$ is indecomposable.

Proof. Thanks to the assumptions, it will hold $(B-A)^{-1}>[0]$. By the previous lemma we have at once the thesis, being $(B-A)^{-1}$ indecomposable.

We note that the indecomposability of the matrix $(B-A)$ is not a sufficient condition for $(B-A)$ to have the connectedness property (it is only a necessary condition for the said property). Consider, e.g., the following numerical example.

$$
(B-A)=\left[\begin{array}{ccc}
1 & -1 & 0 \\
5 & -4 & -3 \\
-2 & 2 & 1
\end{array}\right]
$$

We have

$$
(B-A)^{-1}=\left[\begin{array}{lll}
2 & 1 & 3 \\
1 & 1 & 3 \\
2 & 0 & 1
\end{array}\right]
$$

therefore $(B-A)^{-1} \ngtr[0]$ and it can be seen that $(A, B)$ has not the connectedness property (even if the system is strongly solvable).

For square models $(A, B)$ it is easy to prove that the adjustment property implies that the model is productive: indeed, being the model strongly solvable, we have (Theorem 5) $(B-A)^{-1} \geq[0]$. Let $u=[1,1, \ldots, 1]^{T}$ and let us consider the vector $\bar{x}=(B-A)^{-1} u$. It results $\bar{x}>[0]$, as necessarily $(B-A)$ has at least one positive component in each row (and in each column). Even more can be proved: for any model $(A, B)$, non necessarily square, the adjustment property implies that the model is productive. Indeed, since the model is strongly solvable, for $y=[1,1, \ldots, 1]^{\top}$, there exists an $x \geq[0]$ such that $(B-A) x=y>[0]$, whence the model is productive, by Definition 1. If $(B-A) \in Z$, i.e. for the square matrix $Z=(B-A)$ it holds $z_{i j} \leqq 0, \forall i \neq j$, then $Z^{-1} \geq[0]$ (i.e. the model is all-productive) if and only if $(B-A) \in K$ (in the terminology of Fiedler \& Pták, 1962). There are more than 50 characterizations of the class of the $K$-matrices (among them the famous Hawkins-Simon conditions): see, e.g., Berman and Plemmons (1976), Plemmons (1977), Poole and Boullion (1974), Magnani and Meriggi (1981). Here we give a characterization of the $K$-matrices in terms of the "splitting" $(B-A)$. 
Theorem 9 Let the square matrix $C$ be a Z-matrix; then $C \in K$ if and only if $C$ admits the splitting

$$
C=(B-A)
$$

with $A$ and/or $B$ in the $S$-class, $B$ nonsingular, $B^{-1} A \geqq[0]$ and $\lambda^{*}\left(B^{-1} A\right)<1$.

Proof. First assume that $C \in K$. One of the characterizations of the $K$-class is:

$$
C x>[0], \quad x \geq[0]
$$

admits a solution, i.e. $C \in S$. But then, by choosing $A=[0]$ we have $B=C$ and therefore $B \in K$, which entails $\operatorname{det} B \neq 0, B^{-1} A=[0], \lambda^{*}\left(B^{-1} A\right)=0$. Now we prove the converse implication. Let $C$ satisfy the assumptions of the theorem; then $B^{-1} A \geqq[0]$. We have $B^{-1} C=B^{-1}(B-A)=\left(I-B^{-1} A\right) \in Z$. As $\lambda^{*}\left(B^{-1} A\right)<1$, it holds $B^{-1} A \in K$. Therefore, $\left(I-B^{-1} A\right)^{-1} \geq[0]$, i.e. $\left(B^{-1} C\right)^{-1} \geq[0]$, i.e. $C^{-1} B \geq[0]$ (therefore $C$ is nonsingular). If $B \in S$, then there exists a $q \geqq[0]$ such that $B q>$ [0], i.e. $C C^{-1} B q>$ [0], i.e. $C\left(C^{-1} B q\right)>[0]$. But being $q \geqq[0]$ and $\left(C^{-1} B\right) \geqq[0]$, we have $\bar{q}=C^{-1} B q \geqq[0]$. Therefore, there exists a $\bar{q} \geqq[0]$ such that $C \bar{q}>[0]$, that is $C \in S$. If $A \in S$, then there exists an $x \geqq[0]$ such that $A x>[0]$, i.e. such that $C\left[\left(C^{-1} B\right)\left(B^{-1} A\right)\right] x>[0]$. Therefore, with $\bar{x}=\left(C^{-1} B\right)\left(B^{-1} A\right) x$, being $x \geqq[0], C^{-1} B \geqq[0]$ and $B^{-1} A \geqq[0]$ we have that there exists an $\bar{x} \geqq[0]$ such that $C \bar{x}>[0]$, i.e. $C \in K$.

Obviously, if $A \geqq[0], B \geqq[0]$ and $(B-A) \in Z$, the previous result gives a characterization of an all-productive model. We have, however, to remark that from a purely economic point of view, the case $(B-A) \in Z$ is of scarce interest, unless $B=I$ or $B$ is a diagonal matrix with a positive diagonal.

The conditions which assure that a square matrix $C$, with $c_{i j} \geqq 0, \forall i \neq j$ (i.e. $C$ is a Metzlerian matrix), has a semipositive or a positive inverse, are less known. These conditions are due to Buffoni and Galati (1974). Let us consider a square matrix $C$, of order $n$, nonsingular, indecomposable and such that $c_{i j} \geqq 0, \forall i \neq j$. Denote by $\Delta(i)$ the matrix, of order $(n-1)$, obtained from $C$ by ruling out its $i$-th row and its $i$-th column. The matrix $\Delta(i)$ has a dominant eigenvalue $\mu_{i}$, greater than the real part of any other eigenvalue (see Varga, 1962).

Theorem 10 (Buffoni \& Galati, 1974) Under the above assumptions we have $C^{-1}>[0]$ if and only if the following properties hold together:

(a) For some $i_{0}\left(1 \leqq i_{0} \leqq n\right)$ it holds $\mu_{i_{0}}<0$.

(b) For all $i=1, \ldots, n$, it holds $(\operatorname{det} \Delta(i) / \operatorname{det} C)>0$.

The theory of the "splittings" of a square matrix $C$, i.e. the representation $C=B-A$, has produced several interesting results, especially with reference to iterative methods for the solution of a linear system of equations of the type

$$
C x=b .
$$

However, in several results, it is assumed that one of the two matrices of the splitting (or both) has a semipositive inverse. But in the linear joint production model under consideration, both $A$ and $B$ are nonnegative and, thanks to a result of Johnson (1983), a semipositive square nonsingular matrix has a semipositive inverse only if it is a diagonal matrix or a permutation of a diagonal matrix. Nevertheless, we have the following result.

Theorem 11 Let a square joint production model $(A, B)$ be given, let $B$ be nonsingular with $B^{-1} \geq[0]$. Then:

(i) The model $(A, B)$ is strongly solvable (i.e. all-productive) if and only if $\lambda^{*}\left(B^{-1} A\right)<1$.

(ii) The model $(A, B)$ is strongly solvable and has the connectedness property if and only if $\lambda^{*}\left(B^{-1} A\right)<1$ and the matix $B^{-1} A$ is indecomposable.

(iii) If $(A, B)$ is strongly solvable, then it has the connectedness property if and only if the matrix $B^{-1} A$ is indecomposable.

Proof. (i) The model $(A, B)$ being strongly solvable, thanks to Theorem 5, we have $(B-A)^{-1} \geq[0]$. Moreover, it holds $(B-A)^{-1} B=\left(I-B^{-1} A\right)^{-1} \geq[0]$ and, thanks to a corollary of the Perron-Frobenius theorem: for a semipositive indecomposable matrix $H$, with Frobenius root $\lambda^{*}(H)$, it holds $(\mu I-H)^{-1}>[0]$ if and only if $\mu>\lambda^{*}(H)$ (see, e.g., Debreu \& Herstein, 1953). Therefore, we have $\left(I-B^{-1} A\right)^{-1} \geq[0]$ if and only if $\lambda^{*}\left(B^{-1} A\right)<1$. Being $B^{-1} \geq[0]$ we get $\left(I-B^{-1} A\right)^{-1} B^{-1} \geq[0]$ and we obtain the thesis.

(ii) Being the model $(A, B)$ strongly solvable and having the connectedness property, thanks to $(i)$ it holds $\lambda^{*}\left(B^{-1} A\right)<$ 1 , and thanks to Theorem 6 , the matrix $(B-A)$ is nonsingular, with $(B-A)^{-1}>[0]$. Therefore, $(B-A)^{-1} B=$ 
$\left(I-B^{-1} A\right)^{-1}>[0]$. Therefore, thanks to Lemma 2, the matrix $\left(I-B^{-1} A\right)$ is indecomposable; but being $I$ a diagonal matrix, this property holds if and only if $B^{-1} A$ is indecomposable. The converse case is immediate, thanks to the above quoted corollary of the Perron-Frobenius theorem, applied to the matrix $\left(I-B^{-1} A\right)^{-1}$, with $B^{-1} A$ indecomposable.

(iii) This point follows at once from (i) and (ii).

If we assume Mangasarian's condition (7), equivalent to the implication

$$
p B \geqq[0] \Longrightarrow p A \geqq[0],
$$

it is not possible, even assuming $B$ nonsingular, to get $(B-A)^{-1} \geq[0]$, unless we assume some further properties. With regard to this question, Peris (1991) proves the following result.

Theorem 12 Let a square joint production model (A,B), with B nonsingular, be given. Let the condition (13) be verified and, for all $x \in \mathbb{R}^{n}$ :

$$
\left(\begin{array}{c}
B-A \\
B
\end{array}\right) x \geqq[0] \Longrightarrow x \geqq[0] .
$$

Then the following conditions are equivalent:

(a) The model $(A, B)$ is all-productive;

(b) It holds $\lambda^{*}\left(B^{-1} A\right)<1$;

(c) The model $(A, B)$ is productive (i.e. $(B-A) \in S)$.

We note that (14) is on its turn rather stringent, as it requires that the matrix

$$
C=\left(\begin{array}{c}
B-A \\
B
\end{array}\right)
$$

is of monotone kind, and this holds if and only if $C$ has a nonnegative generalized left inverse (in other words, there must exist an $(n \times 2 n)$ matrix $Y \geqq[0]$ such that $Y C=I)$.

It is however useful to point out that the condition (13) of Mangasarian is a starting point to prove the nonnegativity of the matrix $(B-A)^{-1} A$, a result used by Bapat and others (1995) to obtain results for the generalized eigenvalue problem

$$
A x=\lambda B x, x \geq[0] .
$$

Indeed, supposing $(B-A)$ nonsingular and $B$ nonsingular, we obtain

$$
(B-A)^{-1} A=\left(I-B^{-1} A\right)^{-1} B^{-1} A
$$

and from Mangasarian's condition (7), we obtain $B^{-1} A \geqq[0]$. If (and only if) $\lambda^{*}\left(B^{-1} A\right)<1$, we get $\left(I-B^{-1} A\right)^{-1} \geq$ [0] and therefore $(B-A)^{-1} A \geqq[0]$. The matrix $(B-A)^{-1} A$ has been used also by Schefold (1971, 1978a, 1989 ) in obtaining the existence of a "standard commodity" for the Sraffa model with joint production (Schefold uses a "Sraffa notation", instead of a "Leontief notation"). Moreover, we have to remark that the condition (13) of Mangasarian (even with $B$ nonsingular) and the nonnegativity of the matrix $(B-A)^{-1} A$ are two independent conditions, even for the case of $A$ and $B$ not necessarily nonnegative. Namely, we can have $(B-A)^{-1} A \geqq[0]$ and Mangasarian's condition does not hold; on the other hand, Mangasarian's condition can hold and the matrix $(B-A)^{-1} A$ is not nonnegative (this surely occurs when $\lambda^{*}\left(B^{-1} A\right) \geqq 1$ ).

Take, e.g., the following example:

$$
B=\left[\begin{array}{cc}
1 & 0.1 \\
0.1 & 1
\end{array}\right] ; \quad A=\left[\begin{array}{cc}
0 & 0.5 \\
0.2 & 0.1
\end{array}\right]
$$

Here we have

$$
(B-A)^{-1} A=\frac{1}{0.86}\left[\begin{array}{cc}
0.08 & 0.49 \\
0.2 & 0.15
\end{array}\right],
$$

but Mangasarian's condition (13) is not satisfied. If we take, e.g., the example

$$
B=\left[\begin{array}{cc}
0 & -1 \\
1 & 0
\end{array}\right] ; \quad A=\left[\begin{array}{cc}
-1 & -1 \\
1 & 2
\end{array}\right],
$$


Mangasarian's condition (13) is satisfied, however

$$
(B-A)^{-1} A=\left[\begin{array}{cc}
-1 & -1 \\
-1 / 2 & -1
\end{array}\right] .
$$

Here we have

$$
B^{-1} A=\left[\begin{array}{ll}
1 & 2 \\
1 & 1
\end{array}\right]
$$

and $\lambda^{*}\left(B^{-1} A\right)=1+\sqrt{2}$. This last example is taken from Mehrmann and others (1999).

Finally, we point out that, if the spectral radius of $B^{-1} A, \rho\left(B^{-1} A\right)$, is less than one, it is possible to calculate $(B-A)^{-1}$, by means of a simple generalization of the well-known C. Neumann series. In the case $\left(B^{-1} A\right) \geqq[0]$, obviously we have $\rho\left(B^{-1} A\right)=\lambda^{*}\left(B^{-1} A\right)$.

Lemma 3 Given a matrix $A \in \mathbb{R}^{n \times n}$ it holds

$$
(I-A) \sum_{i=0}^{+\infty} A^{(i)}=I-\lim _{k \rightarrow+\infty} A^{(k)},
$$

where $A^{(i)}$ denotes the $i$-th power of the square matrix $A$.

Proof. We have

$$
(I-A) \sum_{i=0}^{k-1} A^{(i)}=\sum_{i=0}^{k-1} A^{(i)}-A \sum_{i=0}^{k-1} A^{(i)}=\left(I+\sum_{i=1}^{k-1} A^{(i)}\right)-\left(\sum_{i=1}^{k-1} A^{(i)}+A^{(k)}\right)=I-A^{(k)}
$$

and therefore

$$
(I-A) \sum_{i=0}^{+\infty} A^{(i)}=(I-A) \lim _{k \rightarrow+\infty} \sum_{i=0}^{k-1} A^{(i)}=\lim _{k \rightarrow+\infty}(I-A) \sum_{i=0}^{k-1} A^{(i)}=\lim _{k \rightarrow+\infty} I-A^{(k)}=I-\lim _{k \rightarrow+\infty} A^{(k)} .
$$

Theorem 13 Let square matrices $A, B \in \mathbb{R}^{n \times n}$, with $B$ nonsingular and with $\rho\left(B^{-1} A\right)$ spectral radius of $B^{-1} A$, be given Then the following conditions are equivalent:

(a) $\rho\left(B^{-1} A\right)<1$;

(b) $\lim _{k \rightarrow+\infty}\left(B^{-1} A\right)^{(k)}=[0]$, i.e. the matrix $\left(B^{-1} A\right)$ is convergent;

(c) $(B-A)$ is nonsingular and it holds

$$
(B-A)^{-1}=\left(\sum_{i=0}^{+\infty}\left(B^{-1} A\right)^{(i)}\right) B^{-1} .
$$

Proof. $(a) \Longleftrightarrow(b)$. This is a well-known result in matrix calculus, that is usually proved by means of the reduction to the Jordan canonical form. A non-mathematical reader may consult Pasinetti (1977).

$(b) \Longleftrightarrow(c)$. Let us remark first that, thanks to Lemma 3, it holds

$$
\begin{aligned}
(B-A)\left(\sum_{i=0}^{+\infty}\left(B^{-1} A\right)^{(i)}\right) B^{-1} & =B\left[\left(I-B^{-1} A\right) \sum_{i=0}^{+\infty}\left(B^{-1} A\right)^{(i)}\right] B^{-1} \\
& =B\left[I-\lim _{k \rightarrow+\infty}\left(B^{-1} A\right)^{(k)}\right] B^{-1} \\
& =I-B\left[\lim _{k \rightarrow+\infty}\left(B^{-1} A\right)^{(k)}\right] B^{-1} .
\end{aligned}
$$

Being $B$ nonsingular, it follows that $\lim _{k \rightarrow+\infty}\left(B^{-1} A\right)^{(k)}=[0]$ if and only if

$$
B\left[\lim _{k \rightarrow+\infty}\left(B^{-1} A\right)^{(k)}\right] B^{-1}=[0] .
$$


This relation is satisfied if and only if

$$
(B-A)\left(\sum_{i=0}^{+\infty}\left(B^{-1} A\right)^{(i)}\right) B^{-1}=I,
$$

from which the thesis follows.

The above theorem provides an algorithm to compute the inverse of $(B-A)$. Moreover, given the joint production Leontief model

$$
A x+c=B x,
$$

with $A$ and $B$ square, every term of the series which converges to $x=(B-A)^{-1} c$ can be interpreted from the economic point of view, just as for the classical Leontief inverse. See, e.g., Cornwall (1984).

\section{Acknowledgements}

The author thanks an anonymous referee for the many useful suggestions.

\section{References}

Abraham-Frois, G., \& Berrebi, E. (1976). Théorie de la Valeur, des Prix et de l'Accumulation. Paris: Economica.

Abraham-Frois, G., \& Berrebi, E. (1997). Prices, Profits and Rhytms of Accumulation. Cambridge: Cambridge Univ. Press. http://dx.doi.org/10.1017/CBO9780511598937

Bapat, R. B., Olesky, D. D., \& Van Den Driessche, P. (1995). Perron-Frobenius theory for a generalized eigenproblem. Linear and Multilinear Algebra, 40, 141-152. http://dx.doi.org/10.1080/03081089508818429

Berman, A., \& Plemmons, R. J. (1976). Nonnegative Matrices in Mathematical Sciences. New York: Academic Press.

Bidard, C. (1996). All-engaging systems. Economic Systems Research, 8, 323-340. http://dx.doi.org/10.1080/09535319600000025

Bidard, C. (2004). Prices, Reproduction, Scarcity. Cambridge: Cambridge Univ. Press.

Bidard, C., \& Erreygers, G. (1998). The adjustment property. Economic Systems Research, 10, 3-17. http://dx.doi.org/10.1080/09535319800000002

Buffoni, G., \& Galati, A. (1974). Matrici essenzialmente positive con inversa positiva. Bollettino Unione Matematica Italiana, $X, 4^{a}$ serie, $98-103$.

Collatz, L. (1966). Functional Analysis and Numerical Mathematics. New York: Academic Press.

Cornwall, R. R. (1984). Introduction to the Use of General Equilibrium Analysis. Amsterdam: North Holland.

Cottle, R. W., Pang, J. S., \& Stone, R. E. (1992). The Linear Complementarity Problem. New York: Academic Press.

Debreu, G., \& Herstein, I. N. (1953). Nonnegative square matrices. Econometrica, 21, $597-607$. http://dx.doi.org/10.2307/1907925

Fiedler, M., \& Pták, V. (1962). On matrices with non-positive off-diagonal elements and positive principal minors. Czechoslovak Mathematical Journal, 12, 382-400.

Fiedler, M., \& Pták, V. (1966). Some generalizations of positive definiteness and monotonicity. Numerische Mathematik, 9, 163-172. http://dx.doi.org/10.1007/BF02166034

Fujimoto, T., \& Krause, U. (1988). More theorems on joint production. Journal of Economics, 48, 189-196. http://dx.doi.org/10.1007/BF01234610

Gantmacher, F. R. (1959). The Theory of Matrices (2 volls). New York: Chelsea Publishing Co.

Giorgi, G., \& Magnani, U. (1978). Problemi aperti nella teoria dei modelli multisettoriali di produzione congiunta. Rivista Internazionale di Scienze Sociali, 86, 435-468.

Giorgi, G., \& Zuccotti, C. (2013). Some extensions of the K-matrices: characterizations, comparisons and economic applications, submitted. 
Johnson, C. (1983). Sign patterns of inverse nonnegative matrices. Linear Algebra Appl., 55, 69-80. http://dx.doi.org/10.1016/0024-3795(83)90166-0

Kemeny, J. G., Morgenstern, O., \& Thompson, G. L. (1956). A generalization of the von Neumann model of an expanding economy. Econometrica, 24, 115-135.

Kurz, H. D., \& Salvadori, N. (1995). Theory of Production. A Long-Period Analysis. Cambridge: Cambridge Univ. Press.

Lippi, M. (1979). I Prezzi di Produzione. Un Saggio sulla Teoria di Sraffa, Il Mulino, Bologna.

Magnani, U., \& Meriggi, M. R. (1981). Characterizations of K-matrices. In G. Castellani \& P. Mazzoleni (Eds.), Mathematical Programming and Its Economic Applications (pp. 535-547). Milan: F. Angeli.

Manara, C. F. (1968). Il modello di Piero Sraffa per la produzione congiunta di merci a mezzo di merci. L'Industria, 1, 3-18. English translation in Pasinetti (1980).

Mangasarian, O. L. (1971). Perron-Frobenius properties of Ax = $\lambda$ Bx. Journal of Math. Analysis Appl., 36, 86-102.

Mehrmann, V., Olesky, D. D., \& Phan, T. X. T. (1999). Relations between Perron-Frobenius results for matrix pencils. Linear Algebra and Its Appl., 257-269.

Pasinetti, L. L. (1973). The notion of vertical integration in economic analysis. Metroeconomica, $25,1-29$. Reprinted in Pasinetti (1980). http://dx.doi.org/10.1111/j.1467-999X.1973.tb00539.x

Pasinetti, L. L. (1977). Lectures in the Theory of Production. London: Macmillan.

Pasinetti, L. L. (Ed.). (1980). Essays in the Theory of Joint Production. London: Macmillan.

Peris, J. E. (1991). A new characterization of inverse-positive matrices. Linear Algebra and Its Appl., 154-156, 45-58. http://dx.doi.org/10.1016/0024-3795(91)90372-4

Peris, J. E., \& Villar, A. (1993). Linear joint-production models. Econ. Theory, 3, 735-742. http://dx.doi.org/10.1007/BF01210268

Plemmons, R. J. (1977). M-matrix characterizations I-Nonsingular M-matrices. Linear Algebra and Its Appl., 18, 175-188. http://dx.doi.org/10.1016/0024-3795(77)90073-8

Poole, G., \& Boullion, T. (1974). A survey on M-matrices. SIAM Rev., 16, $419-427$. http://dx.doi.org/10.1137/1016079

Schefold, B. (1971). Mr. Sraffa on Joint Production, Diss. Private print, Basel.

Schefold, B. (1976). Relative prices as a function of the profit rate: a mathematical note. Zeitschrift für Nationalökonomie, 36, 21-48.

Schefold, B. (1978a). Multiple product techniques with properties of single product systems. Zeitschrift für Nationalökonomie, 38, 29-53.

Schefold, B. (1978b). On counting equations. Zeitschrift für Nationalökonomie, 38, 253-285.

Schefold, B. (1989). Mr. Sraffa on Joint Production and Other Essays. London: Unwin Hyman.

Sraffa, P. (1960). Production of Commodities by Means of Commodities. Prelude to a Critique of Economic Theory. Cambridge: Cambridge Univ. Press.

Varga, R. S. (1962). Matrix Iterative Analysis. Englewood Cliffs, N.J.: Prentice-Hall.

\section{Copyrights}

Copyright for this article is retained by the author(s), with first publication rights granted to the journal.

This is an open-access article distributed under the terms and conditions of the Creative Commons Attribution license (http://creativecommons.org/licenses/by/3.0/). 